\title{
EDITORIAL
}

\section{A QUOI BON?}

The title question related to the publication of our journal has a number of answer and reflection alternatives, both for its authors and readers alike.

One of the paradoxes of nowadays' IT era is the avalanche of publications governed by the supreme god which is the INTERNET. In order to acquire the basic medical and scientific skills and to upgrade already trained physicians, the Internet has several paradoxical deficiencies:

- The essence is, more often than not, buried under the pile of irrelevant or even false materials;

- The information is, usually, unstructured, hard to categorize in a coherent system and incongruent;

- Original articles end up in the public arena free of charge, after they lose their variety and newsworthiness;

- The ease of access to this "treasure" gives the false impression of knowledge and control when, in fact, they can only be used effectively if they are personally studied.

Based on the above, there is the need to focus on instructing young physicians to think in a clinically medical way, within the mentor-apprentice framework, which is best illustrated by publishing highly visible articles.

Our journal aims at promoting, supporting and encouraging such win-win relations.

In a world continuously tempted by easiness and superficiality, we propose the narrow and bumpy road of hard intellectual work, started as early as student years. In a world of anti-elite levelling tendencies, we support the stringent need to create hierarchies based on authentic values. Last, but not least, in a blasé world, drowned in its own disillusions, we want to re-emphasize the perennial values of creativity, determination, consistency and vision.

Running the risk of falling down the pathetic side, we invite students, physicians and professors to join us in supporting this journal, as part of our belief in the transformative value of authentic science.

Secretary Editor

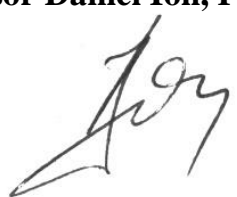

